\title{
ENERGY CONSUMPTION IN A NOVEL ELECTROLYZER HAVING A VERTICAL STACK OF PERFORATED GRAPHITE PLATES AS BIPOLAR ELECTRODES
}

\author{
MASAO SUDOH, HiRoshi KAMEI AND KozO KOIDE \\ Department of Chemical Engineering, Shizuoka University, Hamamatsu 432
}

\begin{abstract}
Key Words: Electrolysis, Bipolar Electrochemical Reactor, Perforated Plate Column, Energy Consumption, Gas-Liquid Flow
\end{abstract}

\begin{abstract}
A novel electrochemical reactor having a vertical stack of perforated graphite plates as bipolar electrodes was investigated by using the electrolysis of sodium bromide. The ratio of Faradaic current to reactor current was explained by an equivalent circuit model which consisted of bypass, sandwiched solution and Faradaic resistances.

Energy consumption for bromine production was influenced by liquid flow regime such as continuous flow or trickle flow and by introducing inert gas countercurrently. Effects of flow regimes of liquid and gas on energy consumption were clarified by the changes of Faradaic current efficiency and the ratio of Faradaic current to total reactor current with the electrode potential difference between the two sides of a bipolar plate.
\end{abstract}

\section{Introduction}

To design an effective electrochemical reactor having bipolar electrodes, considerable efforts are needed for increasing Faradaic current and decreasing shortcircuit and bypass currents. Bipolar packed-bed electrodes were experimentally studied by Yoshizawa et $a l .{ }^{17,18)}$ for waste water treatment and by Kusakabe $e t$ al. ${ }^{14)}$ regarding current paths and electrolysis efficiency. A bipolar rod flow cell was designed and studied by King et al. ${ }^{11,12)}$ on the ratio of Faradaic current to reactor current, and applied by Goodridge et al. ${ }^{1,10)}$ to electrochemical production of propylene oxide. A bipolar trickle bed consisting of carbon Raschig rings or perforated plates was examined by Jansson et al. for organic synthesis, ${ }^{2,7,8,9)}$ pollution control of cyanide-containing effluent ${ }^{5,6)}$ and recovery of metal. ${ }^{4)}$

Recently, Kusakabe et al. ${ }^{13)}$ reported the effect of solution electroconductivity on the overall current efficiency in bipolar packed-bed electrodes. Sasaki et $a l .{ }^{16)}$ discussed the bypass resistance of bipolar disk electrodes with a network model.

The purpose of this study is to clarify the characteristics and the energy consumption of a novel electrochemical reactor having a vertical stack of perforated graphite plates as bipolar electrodes. Experimental studies were made with electrolysis of aqueous solution of sodium bromide in this reactor.

\section{Experimental Apparatus and Procedures}

The bipolar electrochemical reactor, schematically

Received June 9, 1984. Correspondence concerning this article should be addressed to M. Sudoh. shown in Fig. 1, was a cylindrical column made of transparent polyacrylate resin. The dimensions were $5 \mathrm{~cm}$ in inner diameter and $50 \mathrm{~cm}$ in height. Ten perforated plates of graphite, $5 \mathrm{~cm}$ in diameter and $1 \mathrm{~cm}$ thick, were used as bipolar electrodes. Figure 2 shows schematic diagrams of two kinds of graphite plates. The A type plate had 13 holes of $8 \mathrm{~mm}$ diameter and with right triangular pitch, and the B type plate had 12 holes of the same diameter and with square pitch. Five plates of A type and five of B type were arranged by setting A type and B type alternately. The distance $\mathrm{H}$ between two plates was $5 \mathrm{~mm}$ for most experiments. For the experiments shown in Fig. 9, however, the distance was $2 \mathrm{~mm}$ or $1 \mathrm{~cm}$. The top and bottom plates were used as feeder electrodes and connected with a stabilized D.C. power source (NISTAC, NC-12010M). The fifth plate from the bottom was sliced into two equivalent plates, and an insulating plate of polyvinyl chloride resin was inserted between them as shown in Fig. 1. The two separated plates were electrically connected with platinum wire of $0.5 \mathrm{~mm}$ diameter by using electroconductive adhesive of graphite powder and epoxy resin, and the Faradaic current $I_{f}$ through this wire was measured by the galvanometer shown in Fig. 1. Spaces above and below the stack of perforated plates were packed with glass spheres of $1 \mathrm{~cm}$ diameter. An electrolyte was dispersed uniformly on the glass sphere packings with the liquid distributor. The experiments were carried out in continuous-flow and trickle-flow regimes. For continuous-flow operation, nitrogen gas was introduced from the column bottom and flowed countercurrently to liquid flow. For trickle-flow operation, no gas was fed to the reactor. 


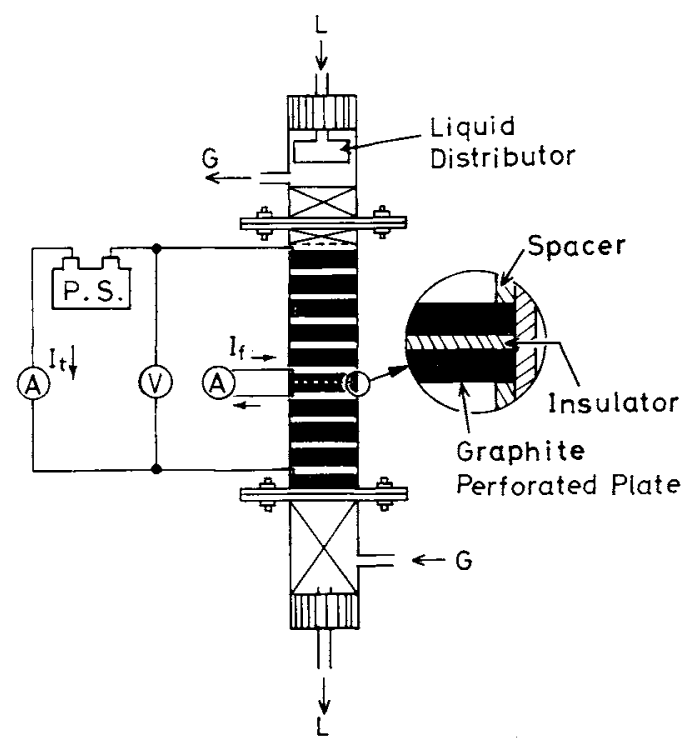

Fig. 1. Schematic diagram of apparatus.

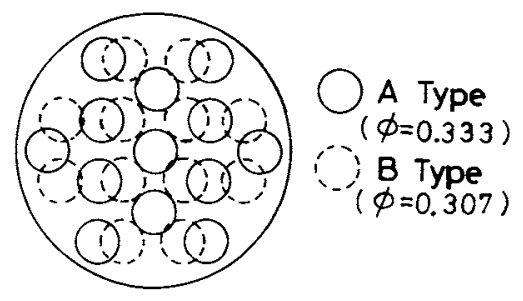

Fig. 2. Detail of perforated graphite plates (A type, $5 \mathrm{~cm}$ in diameter; $8 \mathrm{~mm} \phi \times 13$ holes; $\phi=0.333$. B type, $5 \mathrm{~cm}$ in diameter; $8 \mathrm{~mm} \phi \times 12$ holes; $\phi=0.307$ ).

The electrolyte was a $0.1 \mathrm{~mol} \cdot \mathrm{dm}^{-3}$ aqueous solution of sodium bromide and its temperature was 291-293 K. The electroconductivity of the electrolyte was adjusted with sodium sulfate and measured by a conductivity cell (Toa, CM-30ET). Total resistance of the electrolyte sandwiched between bipolar plates was obtained by measuring A.C. resistance between feeder electrodes at $1 \mathrm{kHz}$. The electrolyte was sampled from the outlet of the reactor, and the concentration of bromine in the electrolyte was determined by iodometric titration in aqueous sulfuric acid.

\section{Equivalent Circuit Model}

The equivalent circuit shown in Fig. 3 consists of the bypass resistance $r_{b}$, the Faradaic resistance $r_{f}$ of a bipolar plate and the sandwiched solution resistance $r_{s}$ between two electrodes. Similar network models for bipolar particles were reported by Robertson et al. ${ }^{15)}$ and King et al. ${ }^{12)}$

Total sandwiched solution resistance $R_{s}$ is given as follows.

$$
R_{s}=\left(N_{B}+1\right) r_{s}
$$

where $N_{B}$ is the number of bipolar plates. Reactor resistance $R_{t}$ is defined by the ratio of voltage and current between feeder electrodes, and is given as

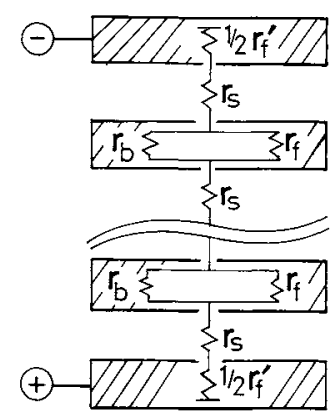

Fig. 3. Equivalent circuit model.

follows.

$$
R_{t} \equiv E / I_{t}=\left(N_{B}+1\right) r_{s}+r_{f}^{\prime}+\left\{N_{B} r_{b} r_{f} /\left(r_{b}+r_{f}\right)\right\}
$$

The Faradaic resistance $r_{f}^{\prime}$ of feeder electrode in Eq. (2) can be ignored because $r_{f}^{\prime}$ is much smaller than $\left(N_{B}+1\right) r_{s}$ or $N_{B} r_{f}$. At low voltage before bipolarity begins, $r_{f}$ is larger than $r_{b}$. Then the total resistance $R_{b}$ is given by the following equation on the basis of Eq. (2).

$$
R_{b} \equiv\left(E / I_{t}\right)_{\circ}=\left(N_{B}+1\right) r_{s}+N_{B} r_{b}
$$

From Eqs. (1)-(3), $r_{s}, r_{b}$ and $r_{f}$ are obtained as follows, respectively.

$$
\begin{aligned}
& r_{s}=R_{s} /\left(N_{B}+1\right) \\
& r_{b}=\left(R_{b}-R_{s}\right) / N_{B} \\
& r_{f}=\left(R_{t}-R_{s}\right)\left(R_{b}-R_{s}\right) /\left\{N_{B}\left(R_{b}-R_{t}\right)\right\}
\end{aligned}
$$

Then the ratio of the Faradaic current $I_{f}$ to the reactor current $I_{t}$ is given by the following equation.

$$
I_{f} / I_{t}=r_{b} /\left(r_{b}+r_{f}\right)=\left(R_{b}-R_{t}\right) /\left(R_{b}-R_{\mathrm{s}}\right)
$$

When the voltage $E$ is applied between feeder electrodes, the difference of electrode potential between the two sides of a bipolar plate, $E_{B}$, is given by using Eq. (2) as follows.

$$
E_{B}=I_{t} r_{b} r_{f} /\left(r_{b}+r_{f}\right)=\left(E-I_{t} R_{s}\right) / N_{B}
$$

$E, I_{t}, I_{f}$ and $R_{s}$ are measured experimentally. $R_{t}$ and $R_{b}$, respectively, are calculated from Eqs. (2) and (3) with the measured values of $E$ and $I_{t} . E_{B}$ is calculated from Eq. (8) with the measured values of $E, I_{t}$ and $R_{s}$.

\section{Faradaic Current Efficiency and Energy Con- sumption}

Electrode reactions at both sides of a bipolar electrode are given below.

Anodic reactions:

$$
\begin{aligned}
& 2 \mathrm{Br}^{-} \rightarrow \mathrm{Br}_{2}+2 \mathrm{e}^{-} \text {at low voltage } \\
& 4 \mathrm{OH}^{-} \rightarrow \mathrm{O}_{2}+2 \mathrm{H}_{2} \mathrm{O}+4 \mathrm{e}^{-} \text {at high voltage }
\end{aligned}
$$

Cathodic reaction:

$$
2 \mathrm{H}^{+}+2 \mathrm{e}^{-} \rightarrow \mathrm{H}_{2}
$$


The Faradaic current efficiency of bromine generation, $C_{e, f}$, is define by the ratio of experimental bromine production to the theoretical value. The latter is given by $\left(N_{B} I_{f} / n \boldsymbol{F}\right)$, if the reactions at feeder electrodes are ignored and only the reaction of Eq. (9) is assumed to occur at the bipolar electrodes.

$$
C_{e, f}=Q_{L} C /\left(N_{B} I_{f} / n F\right)
$$

where $C$ is the bromine concentration at the outlet of the reactor and $Q_{L}$ is the liquid flow rate.

The energy consumption per mole of bromine produced, $E_{C}$, is obtained as follows.

$$
E_{C}=I_{t} E /\left(Q_{L} C\right)
$$

With Eqs. (12) and (13), $E_{C}$ is arranged to the following equation.

$$
E_{C}=n \boldsymbol{F E I} I_{t} /\left(I_{f} N_{B} C_{e, f}\right)
$$

Equation (14) indicates that the following conditions are necessary for decreasing energy consumption.

(i) Bipolarity begins at low voltage $E$.

(ii) $C_{e, f}$ has high value for slightly higher value of $E_{B}$ than bipolarity beginning voltage.

(iii) The ratio of $I_{f} / I_{t}$ is increased by reduction of bypass current.

\section{Results and Discussion}

\subsection{Faradaic current}

Figure 4 shows reactor currents and Faradaic currents at different reactor voltages. Bipolarity of the plates between feeder electrodes began at about $20 \mathrm{~V}$ of reactor voltage. Then Faradaic current began to be observed. The broken lines in Fig. 4 are the Faradaic currents calculated from Eq. (7) with the experimental values of $I_{t}, R_{b}, R_{t}$ and $R_{s}$. The calculated values of $I_{f}$ agree well with the experimental values in the trickleflow regime. In the continuous-flow regime, however, the deviations between calculated and observed values are large at high voltage, where oxygen and hydrogen gases are vigorously evolved by electrolysis of water and the total bypass resistance $R_{b}$ is larger than the value measured from A.C. resistance. Reactor current and Faradaic current for continuous flow are larger than those for trickle flow. This is because the effective surface area of the electrode is reduced for trickle flow.

\subsection{Effects of $U_{L}$ and $U_{G}$ on resistances}

Figure 5 shows $r_{s}, r_{b}$ and $r_{f}$ calculated at $E_{B}=2.5 \mathrm{~V}$ for various flow rates of electrolyte. The gas holdup $\varepsilon_{G}$ in the sandwiched space is estimated by Eq. (15), Bruggemann's equation, ${ }^{3)}$ and is also shown in Fig. 5.

$$
\varepsilon_{G}=1-\left(r_{s 0} / r_{s}\right)^{2 / 3}
$$

where $r_{s 0}$ is the resistance of the sandwiched solution at $\varepsilon_{G}=0$. In the trickle-flow regime, $r_{b}, r_{s}, r_{f}$ and $\varepsilon_{G}$ decrease with increasing $U_{L}$. Gas holdup in the holes

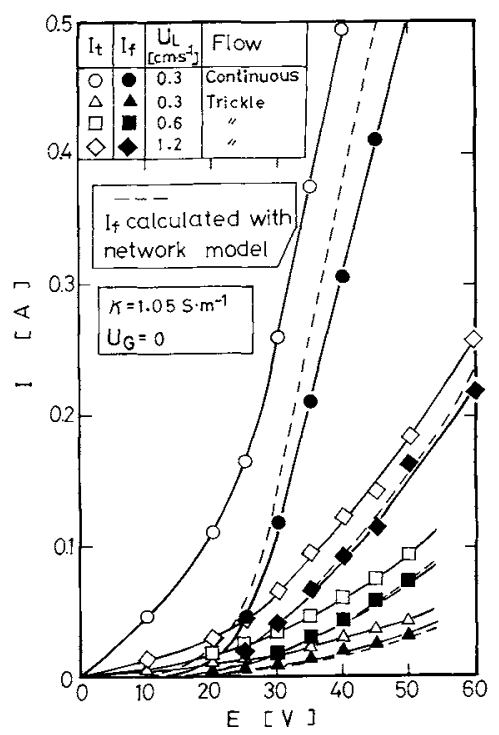

Fig. 4. Relations of reactor current and Faradaic current to reactor voltage.

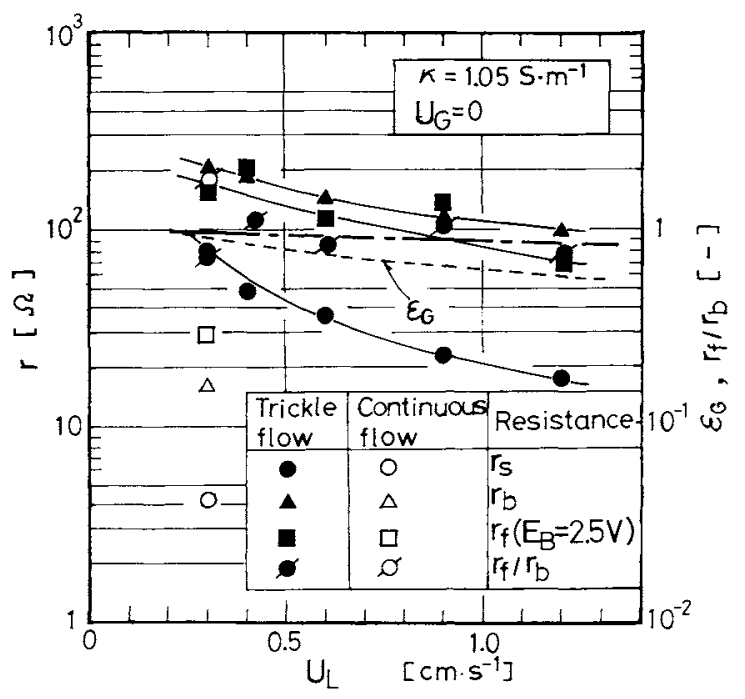

Fig. 5. Resistances of equivalent circuit and $\varepsilon_{G}$ at different liquid velocities.

of a perforated plate is supposed to decrease with increasing $U_{L} . r_{b}$ and $r_{s}$ are dependent on gas holdup. Then, the decreases in $r_{b}$ and $r_{s}$ with increasing $U_{L}$ might be due to the decrease in gas holdup. The decrease in $r_{f}$ with increasing $U_{L}$ might be caused by the enhancement of liquid-solid mass transfer rate of bromide ion. Since the change of $r_{f} / r_{b}$ is small at various $U_{L}$, the change of $I_{f} / I_{t}$ value calculated from Eq. (7) at various $U_{L}$ is expected to be small.

Figure 6 shows that, with increasing $U_{G}$ in the continuous-flow regime, $r_{b}, r_{s}$ and $\varepsilon_{G}$ increase but $r_{f}$ decreases. The increase in $r_{b}$ and $r_{s}$ with increasing $U_{G}$ might be caused by decreased liquid holdup in the holes of the perforated plate and increasing $\varepsilon_{G}$. Meanwhile, the decrease in $r_{f}$ might be caused by increasing liquid-solid mass transfer rate with increasing $U_{G}$. Then, with increasing $U_{G}$, the ratio of $r_{f} / r_{b}$ 


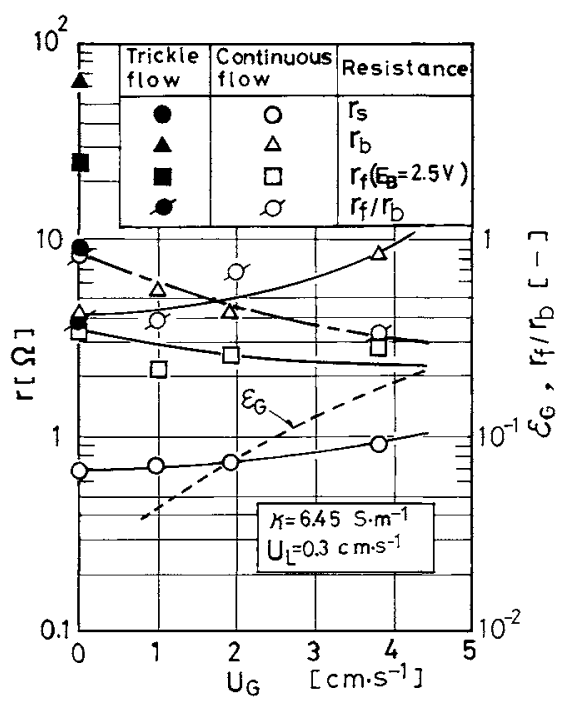

Fig. 6. Resistances of equivalent circuit and $\varepsilon_{G}$ at different gas velocities.

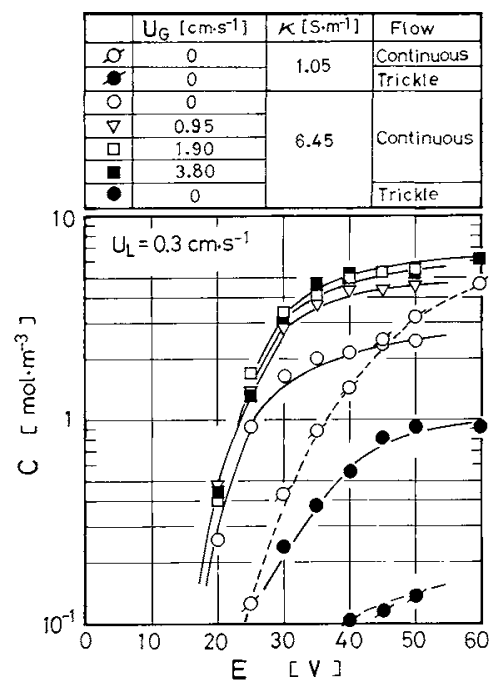

Fig. 7. Relations between outlet concentration of bromine and reactor voltage at different regimes of liquid flow.

decreases and $I_{f} / I_{t}$ calculated from Eq. (7) increases. Therefore, an introduction of gas into the reactor reduces energy consumption in the continuous-flow regime.

Figures 5 and 6 show that $\varepsilon_{G}$ in the trickle-flow regime is much higher than that in the continuousflow regime. Therefore, the resistances in the trickleflow regime become much higher than those in the continuous-flow regime.

4.3 Effects of flow regime and electroconductivity on bromine concentration at reactor outlet

Figure 7 shows the relation between the outlet concentration $C$ of bromine and reactor voltage $E$ in different flow regimes. $C$ increases and approaches a saturated value as reactor voltage increases. In trickle flow, $C$ is lower than that in continuous flow at the same reactor voltage. This is because $I_{f}$ in trickle flow is lower than that in continuous flow, as shown in Fig.

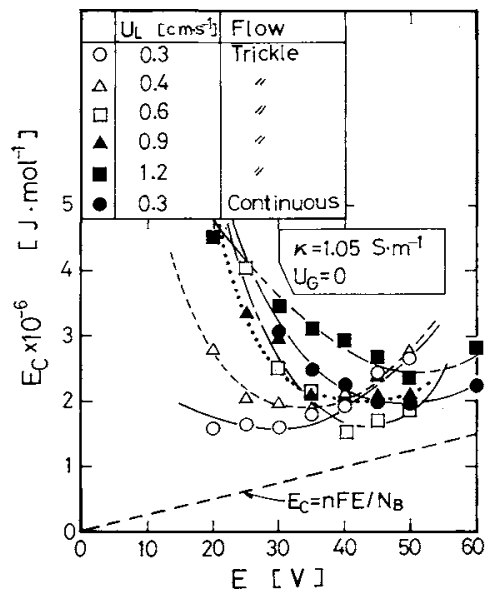

Fig. 8. Relations between energy consumption and reactor voltage at different liquid velocities.

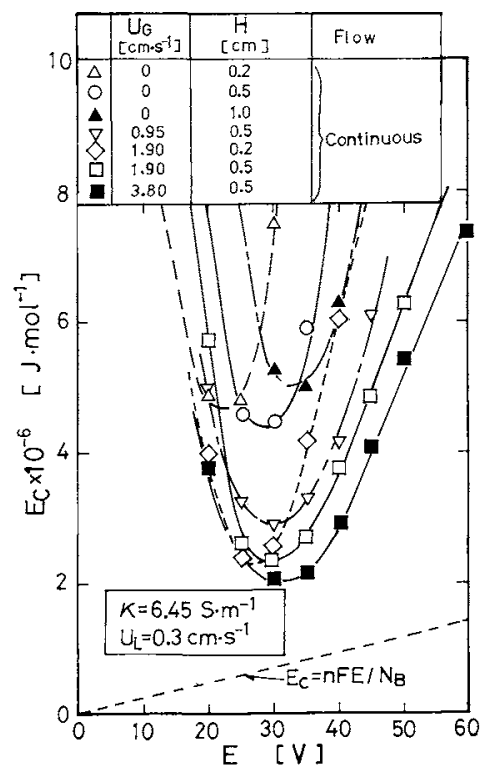

Fig. 9. Relations between energy consumption and reactor voltage at different gas velocities and at different distances between two bipolar perforated plates.

4. In continuous flow with gas introduction, $C$ is higher than that in continuous flow without gas. An introduction of gas increases the liquid-solid mass transfer rate, and then $C$ becomes higher with increasing $U_{G}$.

4.4 Effects of flow regime, $U_{L}, U_{G}$ and electroconductivity on energy consumption

Figure 8 shows the relation between energy consumption and reactor voltage at different liquid velocities. $E_{C}$ has its minimum value at a certain value of $E$. In the trickle-flow regime, the voltage at the minimum value of $E_{C}$ seems to increase with increasing $U_{L}$. At low reactor voltage, $I_{f} / I_{t}$ is much smaller than unity. At high reactor voltage, the value approaches unity but $C_{e, f}$ is low because of water electrolysis. The broken straight lines in Figs. 8, 9 and 11 indicate the theoretical value of $E_{C}$ when $I_{f} / I_{t}=1$ and $C_{e, f}=1$. 
Energy consumption is reduced by increased gas velocity in the continuous-flow regime, as shown in Fig. 9. In comparison with the results in Fig. 8, $E_{C}$ shows a steep curve dependent on $E$, and the minimum value for $E_{C}$ is larger than that in the electrolyte of low conductivity. The latter phenomenon is also observed in the trickle-flow regime.

The effects of distance $H$ between two plates on $E_{C}$ is also shown in Fig. 9. The voltage at the minimum value of $E_{C}$ is lower as the distance becomes shorter. This is because bipolarity begins at lower voltage in the reactor having shorter distance and lower value of $R_{s}$.

Figure 10 shows the effects of $E_{B}$ and the flow regime on $C_{e, f}$ and $I_{f} / I_{t}$. The values of $C_{e, f}$ were calculated from Eq. (12) by using measured values of $C$ and $I_{f}$. The Faradaic current efficiency had its maximum value at $E_{B} \simeq 2.7 \mathrm{~V}$ in the trickle-flow and the continuous-flow regimes. Since anodic reactions are two competitive reactions as described in Eqs. (9) and (10), the values of $C_{e, f}$ were calculated from the ratio of net current for water electrolysis to Faradaic current.

$$
\left(C_{e, f}\right)_{\mathrm{cal} .}=1-I_{f, w} / I_{f}
$$

where $I_{f, w}$ indicates the Faradaic current obtained by water electrolysis in aqueous solution of the same electroconductivity without sodium bromide. The upper part of Fig. 10 shows that the observed values of $C_{e, f}$ agree well with the calculated ones in the range above $2.3 \mathrm{~V}$. In the lower part of Fig. 10, the observed values of $I_{f} / I_{t}$ are compared with those calculated from Eq. (7). Agreement is fairly good in the trickleflow regime. In the continuous-flow regime, there is a large deviation between the values when $E_{B}$ is higher than $2.5 \mathrm{~V}$. As with the explanation of Fig. 4 , this is due to the fact that gas evolving by water electrolysis makes $R_{b}$ higher than the initial value. $I_{f} / I_{t}$ in continuous flow increases with gas velocity, and this is caused by the increase in bypass resistance. $I_{f} / I_{t}$ in trickle flow is larger than that in continuous flow, since $r_{f} / r_{b}$ in trickle flow is smaller than that in continuous flow. As mentioned above, $E_{C}$ is influenced by $C_{e, f}$ and $I_{f} / I_{t}$. Then, $E_{C}$. is assumed to have its minimum value at about $2.7 \mathrm{~V}$ of $E_{B}$.

Figure 11 shows that $E_{C}$ values observed agree well with those calculated from Eq. (14) by using $C_{e, f}$ obtained by Eq. (16) and $I_{f} / I_{t}$ given by Eq. (7). Therefore, $E_{C}$ could be predicted fairly well with the equivalent circuit model, if the contribution of water electrolysis to $C_{e, f}$ was taken into consideration.

Both trickle flow and continuous flow with gas introduction reduced energy consumption. The bromine concentration at the reactor outlet for the latter operation is higher than that in the trickle-flow operation, as shown in Fig. 7. Thus energy con-

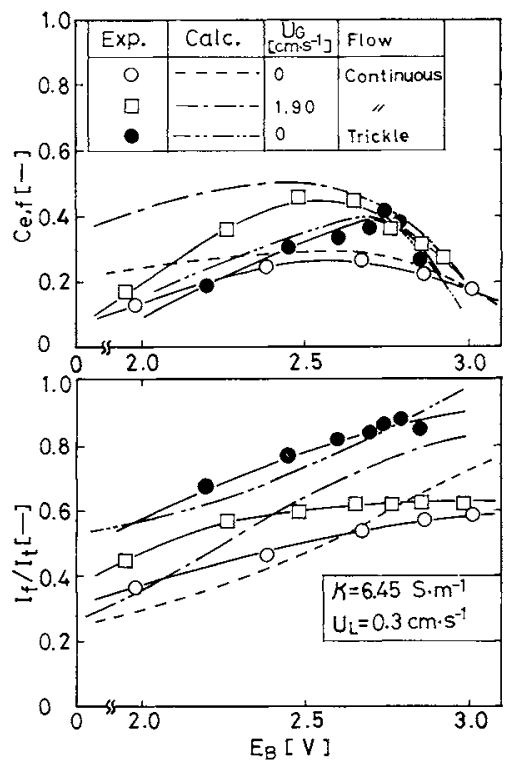

Fig. 10. Relations of Faradaic current efficiency and the ratio of Faradaic current to reactor current at various electrode potential differences between the two sides of a bipolar electrode.

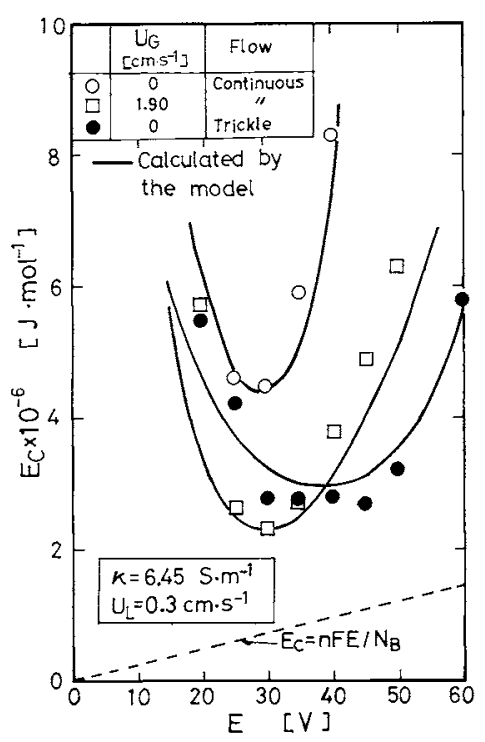

Fig. 11. Comparison between experimental values of energy consumption and values calculated from the model.

sumption and space time yield for bromine generation might be improved by introducing gas into the liquid continuous flow.

\section{Conclusion}

For the continuous-flow regime (countercurrent gas-liquid flow), the energy consumption $E_{C}$ for bromine production was reduced by increasing gas velocity.

For the trickle-flow regime (no gas flow), $E_{C}$ was almost constant at liquid velocity up to $0.9 \mathrm{~cm} \mathrm{~s}^{-1}$ and increased with further increase in liquid velocity.

Low electroconductivity favored the reduction of 
$E_{C}$ for the continuous-flow and the trickle-flow regimes. However, the bromine concentration at the reactor outlet was higher for the higher electroconductivity.

Since water electrolysis was predominant above $2.7 \mathrm{~V}$ of $E_{B}, C_{e, f}$ reached a maximum value and then decreased with increasing $E_{B}, E_{C}$ had its minimum value at about $2.7 \mathrm{~V}$ of $E_{B}$.

The value of $I_{f} / I_{t}$ was predicted fairly well with an equivalent circuit model. The value of $C_{e, f}$ was estimated from the experimental results of water electrolysis. The effects of flow regimes of liquid and gas on $E_{C}$ were clarified by the changes of $I_{f} / I_{t}$ and $C_{e, f}$ with the electrode potential difference between the two sides of a bipolar plate.

An introduction of gas into the liquid continuous flow might be effective in improving energy consumption and space time yield of bromine production.

\section{Nomenclature}

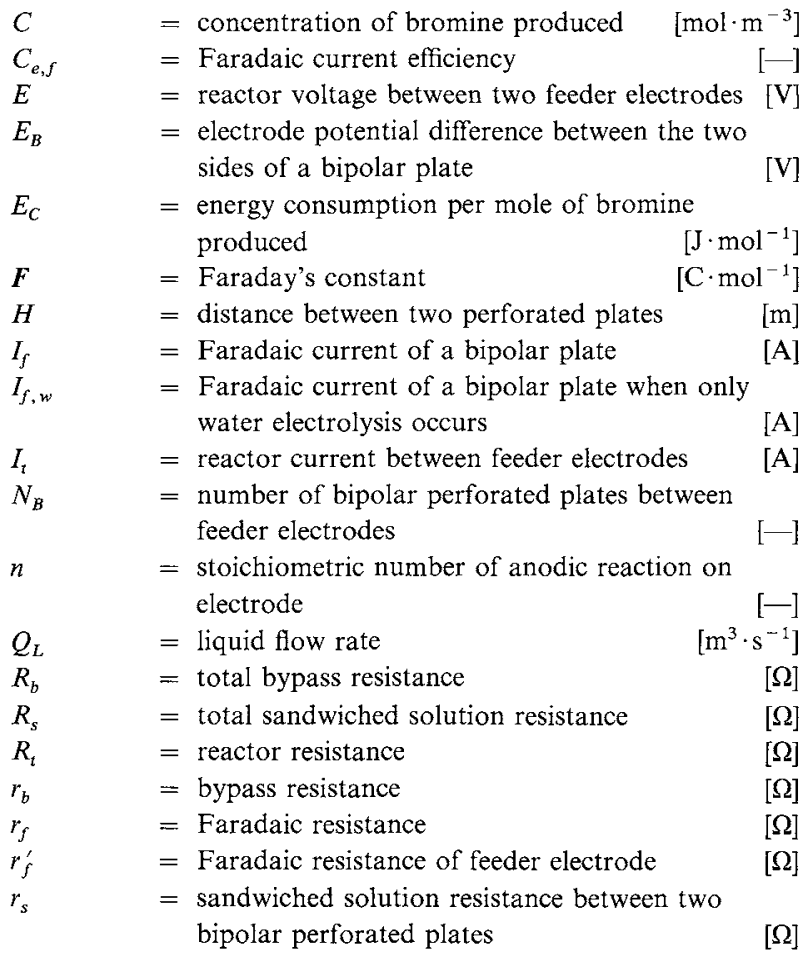

$\begin{array}{llr}r_{s 0} & =r_{\mathrm{s}} \text { for continuous flow when } \varepsilon_{G}=0 & {[\Omega]} \\ U_{G} & =\text { superficial gas velocity } & {\left[\mathrm{m} \cdot \mathrm{s}^{-1}\right]} \\ U_{L} & =\text { superficial liquid velocity } & {\left[\mathrm{m} \cdot \mathrm{s}^{-1}\right]} \\ & & \\ \varepsilon_{G} & =\text { gas holdup in sandwiched solution } & {[-]} \\ \kappa & =\text { electrolyte conductivity } & {\left[\mathrm{S} \cdot \mathrm{m}^{-1}\right]} \\ \phi & & =\text { proportion of free area of a perforated plate }[-]\end{array}$

〈Subscripts〉

- $\quad=$ value at low voltage where bipolarity is not observed

cal. $\quad=$ calculated value

\section{Literature Cited}

1) Bejerano, T., S. Germain, F. Goodridge and A. R. Wright: Trans. Inst. Chem. Eng., 58, 28 (1980).

2) Boussoulengas, A. V., S. Ehdaie and R. E. W. Jansson: Chem. Ind., Oct. 6, 680 (1979).

3) Bruggemann, D. A. G.: Ann. Physik, 24, 636 (1935)

4) Ehdaie, S., M. Fleischmann and R. E. W. Jansson: J. Appl. Electrochem., 12, 59 (1982).

5) El-Ghaoui, E. A. and R. E. W. Jansson: J. Appl. Electrochem., 12, 75 (1982).

6) El-Ghaoui, E. A., R. E. W. Jansson and C. Moreland: J. Appl. Electrochem., 12, 69 (1982).

7) Ellis, K. G. and R. E. W. Jansson: J. Appl. Electrochem., 13, 651 (1983).

8) Ellis, K. G. and R. E. W. Jansson: J. Appl. Electrochem., 13, 657 (1983).

9) Fleischmann, M., J. Ghoroghchian and R. E. W. Jansson: $J$. Appl. Electrochem., 11, 55 (1981).

10) Goodridge, F., C. J. H. King and A. R. Wright: Electrochimica Acta, 22, 347 (1977).

11) King, C. J. H., K. Lister and R. E. Plimley: Trans. Inst. Chem. Eng., 53, 20 (1975).

12) King, C. J. H. and A. R. Wright: Electrochimica Acta, 22, 1135 (1977).

13) Kusakabe, K., T. Kimura, S. Morooka and Y. Kato: J. Chem. Eng. Japan, 17, 293 (1984)

14) Kusakabe, K., S. Morooka and Y. Kato: J. Chem. Eng. Japan, 15, 45 (1982).

15) Robertson, P. M. and N. Ibl: cited from T. Z. Fahidy, Can. J. Chem. Eng., 51, 521 (1973).

16) Sasaki, T. and T. Ishikawa: Denki Kagaku, 52, 318 (1984).

17) Yoshimura, S., A. Katagiri and S. Yoshizawa: Nippon Kagaku Kaishi, 1144 (1978)

18) Yoshizawa, S., Y. Miyazaki and A. Katagiri: Nippon Kagaku Kaishi, 19 (1977). 